\title{
ANGPTL4 supports glutamine metabolism and promotes fatty acid oxidation in nonsmall cell lung cancer cells
}

Song Xiao ( $\sim$ songxiaozjk@hotmail.com )

Hebei North University

\section{Wang Nai-Dong}

Department of Pharmacy, Ji Nan Hospital, Jinan, Shandong, 250000, China

Jin-Xiang Yan

Ningyang No.1 People's Hospital

Long Tian

Radiotherapy Department

Xiu-Rong Lu

Radiotherapy Department

Hong Gao

Beijing Hospatial

Jie-Cheng Yan

Hebei North University

Fei Zhang

Hebei North University

\section{Research}

Keywords: ANGPTL4, NSCLC, glutamine, fatty acid oxidation, aerobic glycolysis

Posted Date: November 13th, 2020

DOl: https://doi.org/10.21203/rs.3.rs-106033/v1

License: (1) (1) This work is licensed under a Creative Commons Attribution 4.0 International License. Read Full License 


\section{Abstract}

Background Angiopoietin-like proteins (ANGPTLs) 4 is key factor in the regulation of lipid and glucose metabolism in metabolic diseases. It also has been demonstrated highly expressed in cancers, but the regulation of energy metabolism on tumor remains to be determined.

Methods Two different NSCLC cells A549 and H1299 were used to investigate the role of ANGPTLs 4 in energy metabolism by tracer technique and seahorse XF technology in ANGPTLs 4 knockdown cell. RNA microarray and specific inhibitors were used in identification targets in overexpression ANGPTLs4 cells.

Results Knockdown ANGPTLs4 could inhibit the energy metabolism and proliferation of NSCLC cells. Knockdown ANGPTLs4 had no significant effect on glycolysis, but affected the glutamine consumption and fatty acid oxidation. Knockdown ANGPTLs4 also significantly inhibited the growth of metastasis and energy metabolism of tumor in mice, but had a weak effect on glycolysis. RNA microarray analysis showed that ANGPTLs4 significantly affected GLS and CPT1. ANGPTLs4 overexpression cells exposed to glutamine deprivation, the effect on cell proliferation and energy metabolism were significantly decreased, but still has difference compared with normal NSCLC cells. ANGPTLs4 overexpression cells treated with GLS and CPT1 inhibitor at the same time, the regulatory effects on cell proliferation and energy metabolism were disappeared.

Conclusions ANGPTLs4 could promote the glutamine consumption and fatty acid oxidation, but not glycolysis, and accelerated the energy metabolism in NSCLC.

\section{Background}

Lung cancer is the most common cause of cancer death in the world, with an estimated 1.6 million deaths per year. From the clinical treatment and biological characteristics, lung cancer is mainly divided into small cell lung cancer and non-small cell lung cancer. About $85 \%$ of patients collectively referred to as non-small cell lung cancer (NSCLC) [1]. Abnormal energy metabolism is the characteristic of tumor[23]. The classical example of a reprogrammed metabolic pathway in cancer is the Warburg effect or aerobic glycolysis [4]. Cancer cells undergo metabolic reprogramming also upregulation of de novo lipid synthesis and glutaminolysis [5-6]. Tumor cells recombine their metabolism, including changes in glucose and fat metabolism, to meet the requirements of cell biosynthesis and cell function.

Angiopoietin-like protein 4 (ANGPTL4) is a multifunctional cytokine that is involved in both angiogenesis and metastasis[7]. ANGPTL4 exhibits structural similarity to the multifunctional angiopoietins, which are involved in glucose homeostasis, lipid metabolism, angiogenesis, inflammation, and tumor progression and metastasis[8-10]. ANGPTL4 serves an important role in the tumor microenvironment, especially that which is hypoxia-induced. Therefore, in recent years, the research on ANGPTL4 in tumor function has been found that activation of ANGPTL4 in lung cancer cells by HIF-a can promote the proliferation of tumor cells[11]. ANGPTL4-deficiency by genetic knockdown or treatment with a neutralizing antibody led to a significant reduction in obesity-induced angiogenesis and tumor growth[12]. However, how ANGPTL4 
participates in the energy metabolism of tumor cells, especially its mechanism in NSCLC, remains to be elucidated.

\section{Materials And Methods}

\section{Cells culture}

Two NSCLC cell lines (A549, H1299) was purchased from the Cell Bank of the Chinese Academy of Sciences (Shanghai, China). NSCLC cells were maintained in DMEM (Gibco, Grand Island, NY, USA) supplemented with $10 \%$ fetal bovine serum (FBS; Gibco), penicillin $(100 \mathrm{U} / \mathrm{ml})$ and streptomycin $(100 \mu \mathrm{g} / \mathrm{ml})$ (Beyotime Biotech, Haimen, China) in humidified air at $37^{\circ} \mathrm{C}$ with $5 \% \mathrm{CO}_{2}$.

\section{Knockdown/ overexpression of ANGPTL4 in NSCLC}

To knock down ANGPTL4 expression, siRNA (Thermo Fisher Scientific) was transfected using RNAiMax (Invitrogen, Carlsbad, CA) according to the manufacturer's recommendations. The sequence of the siRNAs was referred to existing reports. ${ }^{13}$ Control cells were transfected with scrambled siRNA (Thermo Fisher Scientific). Overexpression of ANGPTL4 Cells were transfected in A549 cells (Santa Cruz, USA) to increased the level of ANGPTL4(S-5'-TCTCTCACCGGGTATGAGCGGTGCTCCGACGGCC-3', AS-5'GTGTCTTAATTAACTAGGAGGCTGCCTCTGCTGC-3'). Full-length cDNA encoding human ANGPTL4 were cloned into the vector plasmid (Shanghai Genechem Co., Ltd).

\section{Glutamine-deprivation cell model}

Glutamine deprivation cell model was established, remove normal DMEM, and wash normal cells or transfect cells with PBS. Adding DMEM (ThermoFisher Science, A14431) which without glutamine. In addition, 10\% DFBS (Gemini Bio Products, Sacramento, California, USA) was added to the medium. The cells were cultured in this medium for 24 hours. After the cells adapted to the medium, they were passaged normally and continued to be used.

\section{Cell proliferation assay with cell counting Kit-8 (CCK-8)}

96-well plate was used to implant cells. The initial cell concentration was 5000 cells/pore, and the cell proliferation activity was measured by CCK-8 (Beyotime Biotech, Haimen, China). CCK-8 reagent was added to the pore after $24,48,72$ and 96 hours of cell growth. The cells were cultured in cell culture box for 1.5 hours. Absorption was measured at $450 \mathrm{~nm}$ to investigate the cell proliferation activity.

\section{Animal and tumor model}

Six- to eight-week male nude mice were obtained from the Hebei Medical University experimental animal center. The mice were housed in a specific pathogen-free facility with free access to normal chow and water. Individual nude mice were inoculated subcutaneously with A549 cells $\left(1 \times 10^{6}\right.$ cells in $0.1 \mathrm{ml}$ of PBS) on the right leg. When a tumor volume reached $\sim 50 \mathrm{~mm}^{3}$, individual mice were randomized and treated intravenously with $0.1 \mathrm{ml}$ of $5 \%$ glucose solution through the caudal vein as a vehicle, $10 \mu \mathrm{g}$ 
liposomeencapsulated pshRNA-Con or pshRNA- ANGPTL4. Their body weights and tumor growth were measured every day, and the tumor volumes $(V)$ were calculated using the formula: $V=a \times b^{2 / 2}$, where $a$ and $b$ are the largest and smallest tumor diameter, respectively. The mice were sacrificed 24 hours after the final treatment. Each experiment was performed twice.

\section{ATP and lactate production}

Normal or transfected cells were inoculated into 6-well plates. After washing with PBS, the cells were digested with $0.25 \%$ trypsin and the protein content was quantified by BCA kit. The ATP (Biovision, K354100 ) and lactic acid (Biovision, K462) content in cells and tumor model in mice were measured by spectrophotometer and commercial kit.

\section{Isotope tracing metabolomics of glutamine and FAO}

The isotope tracing metabolomics was used. We used DMEM medium that was glucose- and glutamine-

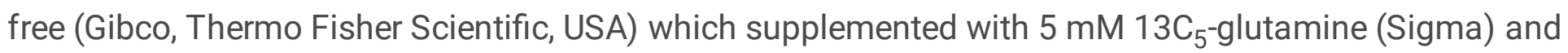
${ }^{13} \mathrm{C} 16$-palmitate. Following 16 hours of incubation, cells were harvested and the metabolites extracted with ice cold methanol and measured by LC/MS as described

\section{Gene detection and western blotting}

Total RNA was extracted from cells using TRIzol reagent (Thermo Fisher Scientific). The purity of RNA was determined by NanoDrop ND-1000 spectrophotometer (Thermo Scientific) qPCR was conducted using the appropriate primers and a Bio-Rad CFX96 system with SYBR green to determine the mRNA expression levels of genes incells. GAPDH was used as endogenous controls and further analyzed by the $2^{\Delta}$ DDCT method. All the primers used for qPCR are listed in the supplement data. Statistical analysis was performed on the fold change

Total proteins were isolated from A549 and H1299 cells and dissolved in Ripa buffer which containing protease inhibitor (sigma, USA). The total protein concentration was determined by BCA analysis kit (Rockford Pierce, Illinois, USA). Total protein samples (30 mg) were analyzed by $10 \%$ SDS-PAGE gel and transferred to PVDF membrane. After the blocking procedure, the cell membrane was incubated overnight with primary antibody and secondary antibodies. Then, visualized in Imager (Bio-Rad) using ECL system (Thermo Fisher Scientific, 34095). The following antibodies were used: ANGPTL4 (1:5000, abcam, MA, USA), GLS (1:1000, abcam, MA, USA),CPT1 (1:1000, abcam, MA, USA), GAPDH (1:5000, abcam, MA, USA)

\section{Statistical Analyses}

All experimental results were analyzed by SPSS 17.0 software and expressed as mean \pm SEM. Standard ANOVA procedures followed by multiple pairwise comparison adjusted with Bonferroni corrections were performed for cell viability assays. Unpaired Student's t-tests were used to analyse all the other results. Significance was considered at $p<0.05$. 


\section{Results}

\section{ANGPTL4 perform OXPHOS but not aerobic glycolysis}

In order to investigate the effect of ANGPTL4 on NSCLC cells, A549 and H1299 cells were used to established ANGPTL4-knockdown NSCLC cells, respectively. The results showed that the cell proliferation of NSCLC cells decreased significantly after ANGPTL4 knockdown compared to normal NSCLC cells (Fig. 1A, B). At the same time, ATP were also examined in A549 and H1299 cells. The content of ATP decreased significantly in ANGPTL4 knockdown cells (Fig. 1C).

Knockdown ANGPTL4 significantly reduced the energy metabolism level of cells. The effect of ANGPTL4 on energy metabolism of NSCLC cells in glycolysis or oxidative phosphorylation (OXPHOX) were investigated. Therefore, to understand the real-time state of glycolysis and the mitochondrial oxygen consumption rate (OCR), the Seahorse XF technology was utilized and measured the OCR and extracellular acidification rate (ECAR) ratios in ANGPTL4 knockdown cells. Following addition of glucose, the glycolytic rate was no significantly different between ANGPTL4 knockdown cell and normal NSCLC cells. Moreover, glucose consumption and lactate level had no significant change in all ANGPTL4 knockdown cell lines (Fig. 2A). In contrast, the results showed that oxygen consumption rate (OCR) in ANGPTL4 knockdown cell significantly decreased compared to normal cells(Fig. 2B). The results show that the expression of ANGPTL4 could promote the energy synthesis of NSCLC cells, perform OXPHOS but not aerobic glycolysis.

\section{ANGPTL4 promotes glutamine metabolism and fatty acid oxidation}

Seahorse XF mito fuel flex test showed ANGPTL4 perform OXPHOS but not aerobic glycolysis. Glutamine or fatty acids as an alternative source of energy in OXPHOS. To better understand ANGPTL4-specific metabolic mechanisms, we analyzed the metabolites in vitro ${ }^{13} \mathrm{C}$ labeling of ANGPTL4 knockdown and control cells. ANGPTL4 knockdown and control cells incubated for $4 \mathrm{~h}$ in the presence of $5 \mathrm{mM} 13 \mathrm{C}_{5^{-}}$ glutamine (Sigma) or $13 \mathrm{C}_{16}$-palmitate. ANGPTL4 knockdown could significantly reduce the related substances that enter the TCA cycle of mitochondria through glutamine metabolism. The related products of glutamine metabolism isotope-encoded $a-K G(M+5)$, aspartate $(M+4)$ and malate $(M+4)$ was significantly decreased in ANGPTL4 knockdown cells (Fig. 3A). ANGPTL4 metabolize derive a much greater fraction of total acetyl-CoA from $\left[13 \mathrm{C}_{16}\right]$-palmitate. After knocking out ANGPTL4, the content of $\left[13 \mathrm{C}_{16}\right]$-acetyl-CoA and [13C $\left.\mathrm{C}_{16}\right]$-acetylcarnitine decreased significantly (Fig. 3B).

The results of RNA microarray analysis showed that knockdown ANGPTL4 has certain effects on fatty acid oxidation, glutamine metabolism and glycolysis pathway gene expression (supplement data1,Table S1). The gene expression of CPT1 and GLS was significantly decreased(supplement data10Figure S1)., respectively. The results of protein quantification were consistent with those of gene chip (Fig. 3C). 
To determine the effect of ANGPTL4 silencing on the NSCLC energy metabolism in vivo, nude mice were inoculated subcutaneously with $1 \times 10^{6}$ A549 cells, and when the implanted tumors grew at $50 \mathrm{~mm}^{3}$ in one dimension, the mice were randomized and treated intravenously with vehicle as controls, pshRNAcontrol, pshRNA- ANGPTL4, respectively. The growth of implanted tumors in individual mice were monitored longitudinally, and growth of implanted tumors in different groups of mice were indistinguishable at individual time points (Fig. 4A). Control plasmid did not affect the growth of implanted tumors in mice, but treatment with pshRNA- ANGPTL4 significantly inhibited the growth of implanted tumors in mice. The content of glutathione and acetyl CoA in the tumor of nude mice were detected, but had little effect on the content of lactate. The results showed that the content of glutathione and acetyl $\mathrm{COA}$ in the tumor of nude mice decreased significantly after administration of pshRNAANGPTL4(Fig. 4B).

\section{ANGPTL4 upregulated glutaminase and acetyl-CoA synthesis in NSCLC cells promotes glutamine metabolism and fatty acid oxidation}

To examine whether the ANGPTL4 mediated cell growth through glutamine and fatty acid oxidation dependent, we cultured ANGPTL4 overexpression cells in medium with or without glutamine, and found that the effects on cell proliferation activity of NSCLC cells was strikingly decreased in glutamine-free medium compared to glutamine medium. However, there was still statistical differences in glutamine-free medium compared with normal cells(Fig. 5A). The effect of ANGPTL4 on OXPHOS was determined by giving CPT1 or GLS inhibitors. The results showed that ANGPTL4 overexpression significantly promoted the OXPHOS in NSCLC cells, but significantly decreased when GLS inhibitor or GLS inhibitors was given, and disappeared in combined with both GLS inhibitor and GLS inhibitors (Fig. 5B). The effect of ANGPTL4 on glutamine metabolism and fatty acid oxidation of cells was also further investigated by giving CPT1 or GLS inhibitors. The results showed that when GLS inhibitor was given, the regulatory effect of a on glutamine metabolites disappeared, the related products of glutamine metabolism isotopeencoded $a-K G(M+5)$, aspartate $(M+4)$ and malate $(M+4)$ was significantly decreased. [13C 16 -acetyl$\mathrm{CoA}$ and $\left[13 \mathrm{C}_{16}\right]$-acetylcarnitine decreased significantly when CPT1 inhibitor was given, the regulatory effect on fatty acid metabolites disappeared (Fig. 6).

\section{Discussion}

The reorganization of energy metabolism is the power source of tumor cell proliferation. Metabolic reprogramming has taken place in sugar metabolism, amino acid metabolism, lipid metabolism and other aspects[14]. Glycolysis is the main feature of energy metabolism in tumor cells. Pyruvate produced by glycolysis is not coupled with TCA cycle in mitochondria, but converted into lactic acid to produce ATP [16-17]. Besides glycolysis, the mitochondrial TCA cycle is another source of energy for tumor cells. Glutamine is one of the important raw materials for TCA cycle of tumor cells.[18]. After glutamine is converted into glutamic acid, it is catalysed to generate $a$ - ketoglutarate $(a-K G)$ to enter the tricarboxylic acid cycle to provide carbon source. The pathway of fatty acid and cholesterol synthesis in tumor is active, including acetyl CoA carboxylase (ACC), fatty acid synthetase (FASN) [19-20]. In addition, lipid 
metabolism is also an important energy source for tumor cells. It was found that the high expression of genes related to lipid uptake and transport, lipid synthesis and intracellular distribution, and fatty acid oxidation were involved in the high metastatic tumor cells. ${ }^{21}$

The angiopoietin-like 4 (ANGPTL4) protein belongs to a superfamily of secreted proteins structurally related to factors modulating angiogenesis known as angiopoietins. This protein family includes eight members encoded by eight genes (ANGPTL1-8) identified in humans and mice[22]. Previous studies have found that ANGPTL4 is highly expressed in breast cancer, colorectal cancer, prostate cancer, liver cancer, kidney cancer and other tumors, and participates in the regulation of tumor growth and development, redox reaction, angiogenesis and metastasis and other biological functions. ANGPTL4 also participates in lipid metabolism and regulates cell energy metabolism[23]. ANGPTL4 deficiency in macrophages results in ER stress due to the cell-intrinsic reprogramming of fatty-acid metabolism[24]. ANGPTL4 has surfaced as principal regulators of plasma lipid metabolism by functioning as potent inhibitors of lipoprotein lipase in cardiovascular disease[25].

Through gene knockdown technology, we found that after knockdown of ANGPTL4 in different NSCLC cells, the cell proliferation activity and energy metabolism level decreased significantly, which indicated that ANGPTL4 could affect the proliferation ability of NSCLC cells by participating in energy metabolism. With the seahorse XF technology, the differences of oxidative phosphorylation and glucose mediated cell acidification metabolism in NSCLC cells after ANGPTL4 knockdown were analyzed. The experimental results showed that compared with the characteristic glycolysis of tumor cells, ANGPTL4 had more significant effect on oxidative phosphorylation, ANGPTL4 was more involved in oxidative phosphorylation of tumor cells, but had less effect on glycolysis.

The main raw materials of oxidative phosphorylation of tumor cells are glutamine and fatty acid $\beta$ oxidation. The research shows that ANGPTL4 was involved in the metabolism regulation of the body. At present, there were many researches about a participating in the lipid metabolism of the body. Angiopoietin-like protein (ANGPTL)4 regulates plasma lipids, ANGPTL4 deficiency increases lipid uptake and respiration in macrophages[26]. ANGPTL-4 presented a negative correlation with BMI, waist circumference, weight, insulin[27]. It is found that ANGPTL-4 is involved in the regulation of lipid metabolism in metabolic syndrome, obesity, diabetes and cardiovascular disease[28]. However, there are few studies on the regulation of lipid metabolism by ANGPTL-4 in tumor cells. Using isotope labeling technique, we investigated the effect of knockdown ANGPTL-4 on glutamine metabolites and fatty acid oxidation in NSCLC cells. The results showed that the content of glutamine metabolites a-KG(M+5), aspartate $(M+4)$ and malate decreased significantly after knocking out ANGPTL-4. Glutamine is converted into glutamic acid, it is catalysed to generate $a$ - ketoglutarate $(\alpha-K G)$ participate in mitochondrial TCA cycle. The main sources of acetyl CoA are glucose, lipid metabolism in normal cell. Previous studies have found that a has no significant effect on the glycolysis of NSCLC. The results of isotope labeling showed that ANGPTL-4 significantly affected the production of acetyl CoA from lipid metabolism. In order to further clarify the effect of ANGPTL-4 on energy metabolism of tumor cells, implanted tumors animal model was established, nude mice were inoculated subcutaneously with A549 
cells. When pshRNA- ANGPTL4 was given to mice, the expression of ANGPTL4 was significantly reduced, while the tumor was significantly reduced and the tumor energy metabolism was significantly reduced. The content of glutathione and acetyl CoA in the tumor of nude mice were decreased significantly after administration of pshRNA- ANGPTL4, but had little effect on the content of lactate. RNA microarray analysis showed that knockdown ANGPTL4 has certain effects on fatty acid oxidation, glutamine metabolism and glycolysis pathway gene expression. The gene expression of CPT1 and GLS was significantly decreased. To examine whether the ANGPTL4 mediated cell growth through glutamine and fatty acid oxidation dependent though CPT1 and GLS. ANGPTL4 overexpression cells were cultured in medium with or without glutamine, and found that the effects on cell proliferation activity of NSCLC cells was strikingly decreased in glutamine-free medium compared to glutamine medium. However, compared with normal cells, there are still statistical differences in glutamine-free medium. In ANGPTL4 overexpression cells,the regulatory effect on glutamine metabolism and fatty acid metabolites disappeared, there was no significant difference in the proliferative activity between ANGPTL4 overexpression cells and normal NSCLC cells.

In conclusion, ANGPTL4 is involved in glucose metabolism and lipid metabolism, which can affect the occurrence and development of many chronic diseases, In this study, we found that ANGPTL4 can affect the energy metabolism of NSCLC cells and then the proliferation of cells, and the main mechanism of its influence on the energy metabolism of NSCLC cells is to affect CPT1 and GLS, then regulated glutamine metabolism and fatty acid oxidation, rather than glycolysis.

\section{Declarations}

\section{Ethics approval and consent to participate}

Not applicable

\section{Consent for publication}

Not applicable

\section{Availability of data and materials}

The datasets during and/or analysed during the current study available from the corresponding author on reasonable request.

\section{Competing interests}

The authors declare that there are no conflicts of interest

\section{Funding}

This study was funded by the Subject of ZHANGJIAKOU $(1821155 \mathrm{H})$ 


\section{Acknowledgment}

Not applicable

\section{Authors' contributions}

Song xiao : perception and design; participation in the whole work; final approval of the version to be published; Wang Nai-dong, Yan jin-xiang,Tian long, Lu xiu-rong, Gao hong, Yan jie-cheng, Zhang fei : Participation in the whole work; data analysis;

\section{References}

1. Arbour KC. Systemic Therapy for Locally Advanced and Metastatic Non-Small Cell Lung Cancer: A Review. JAMA. 2019;322:764-774.

2. DeBerardinis RJ, Chandel NS. Fundamentals of cancer metabolism. Sci Adv. 2016;2:e1600200.

3. Lane AN, Higashi RM, Fan TW. Metabolic reprogramming in tumors: Contributions of the tumor microenvironment. Genes Dis. 2019;7:185-198

4. Bose S, Le A. Glucose Metabolism in Cancer. Adv Exp Med Biol. 2018;1063:3-12.

5. Zong WX, Rabinowitz JD, White E. Mitochondria and Cancer. Mol Cell. 2016;61:667-676.

6. Zecchini V, Frezza C. Metabolic synthetic lethality in cancer therapy. Biochim Biophys Acta Bioenerg. 2017;1858:723-731.

7. Masuko K. Angiopoietin-like 4: A molecular link between insulin resistance and rheumatoid arthritis. J Orthop Res. 2017;35:939-943.

8. Olshan DS, Rader DJ. Angiopoietin-like protein 4: A therapeutic target for triglycerides and coronary disease? J Clin Lipidol. 2018;12:583-587.

9. Cinkajzlová A, Mráz M, Lacinová Z, et al. Angiopoietin-like protein 3 and 4 in obesity, type 2 diabetes mellitus, and malnutrition: the effect of weight reduction and realimentation. Nutr Diabetes. 2018;8:21.

10. Tan MJ, Teo Z, Sng MK, et al. Emerging roles of angiopoietin-like 4 in human cancer. Mol Cancer Res. 2012;10:677-88.

11. Hata S, Nomura T, Iwasaki K, et al. Hypoxia-induced angiopoietin-like protein 4 as a clinical biomarker and treatment target for human prostate cancer. Oncol Rep. 2017;38:120-128.

12. Kolb R, Kluz P, Tan ZW, et al. Obesity-associated inflammation promotes angiogenesis and breast cancer via angiopoietin-like 4. 2019;38:2351-2363.

13. Pal M, Tan MJ, Huang RL, et al. Angiopoietin-like 4 regulates epidermal differentiation. PLoS One. 2011;6:e25377

14. Sun L, Suo C, Li ST, et al. Metabolic reprogramming for cancer cells and their microenvironment: Beyond the Warburg Effect. Biochim Biophys Acta Rev Cancer. 2018;1870:51-66. 
15. Faubert B, Solmonson A, DeBerardinis RJ. Metabolic reprogramming and cancer progression. 2020;368(6487). pii: eaaw5473.

16. Gill KS, Fernandes $P, O$ 'Donovan TR, et al. Glycolysis inhibition as a cancer treatment and its role in an anti-tumour immune response. Biochim Biophys Acta. 2016;1866:87-105.

17. Ganapathy-Kanniappan S, Geschwind JF. Tumor glycolysis as a target for cancer therapy: progress and prospects. Mol Cancer. 2013;12:152.

18. Akins NS, Nielson TC, Le HV. Inhibition of Glycolysis and Glutaminolysis: An Emerging Drug Discovery Approach to Combat Cancer. Curr Top Med Chem. 2018;18:494-504.

19. Li Z1, Zhang H. Reprogramming of glucose, fatty acid and amino acid metabolism for cancer progression. Cell Mol Life Sci. 2016;73:377-92.

20. Röhrig F, Schulze A. The multifaceted roles of fatty acid synthesis in cancer. Nat Rev Cancer. 2016;16:732-749.

21. Tang NT, D Snook R, Brown MD, et al. Fatty-Acid Uptake in Prostate Cancer Cells Using Dynamic Microfluidic Raman Technology. 2020 ,25(7). pii: E1652.

22. Fernández-Hernando C, Suárez Y. ANGPTL4: a multifunctional protein involved in metabolism and vascular homeostasis. Curr Opin Hematol. 2020;27:206-213.

23. Aryal B, Price NL, Suarez Y, et al. ANGPTL4 in Metabolic and Cardiovascular Disease. Trends Mol Med. 2019;25:723-734.

24. Al Shawaf E, Abu-Farha M, Devarajan S, et al. ANGPTL4: A Predictive Marker for Diabetic Nephropathy. J Diabetes Res. 2019;2019:4943191.

25. Chen RM, Yuan X, Ouyang Q, et al. Adropin and glucagon-like peptide-2 are associated with glucose metabolism in obese children. World J Pediatr. 2019;15:565-571.

26. Aryal B, Price NL, Suarez Y, et al. ANGPTL4 in Metabolic and Cardiovascular Disease. Trends Mol Med. 2019;25:723-734.

27. Ding $S$, Wu D, Lu Q, et al. Angiopoietin-like 4 deficiency upregulates macrophage function through the dysregulation of cell-intrinsic fatty acid metabolism. Am J Cancer Res. 2020;10:595-609.

28. Dewey FE, Gusarova V, O'Dushlaine C, et al. Inactivating Variants in ANGPTL4 and Risk of Coronary Artery Disease. N Engl J Med. 2016;374:1123-33.

\section{Figures}


A
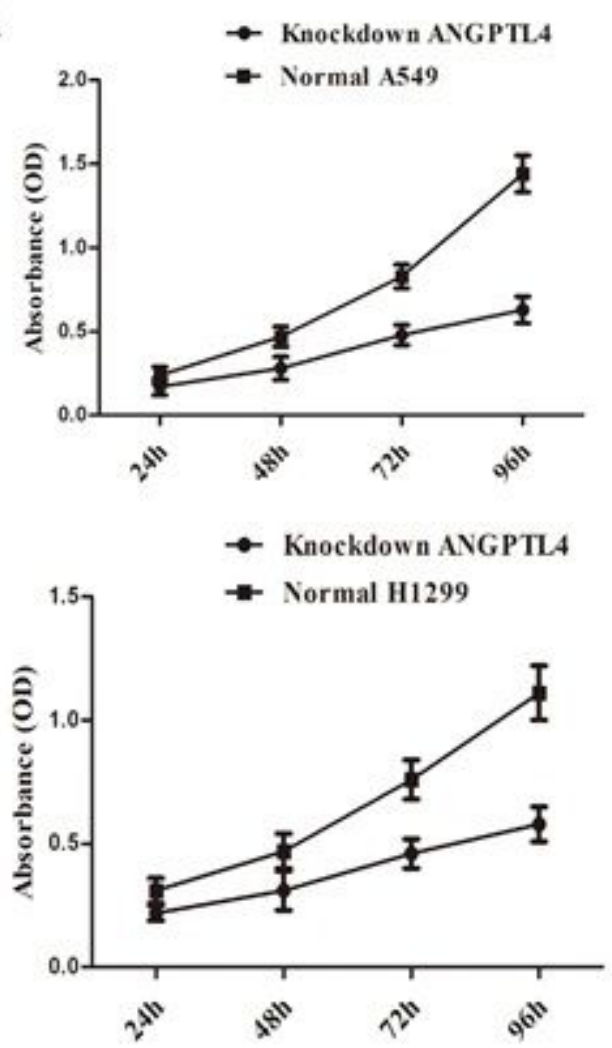

B
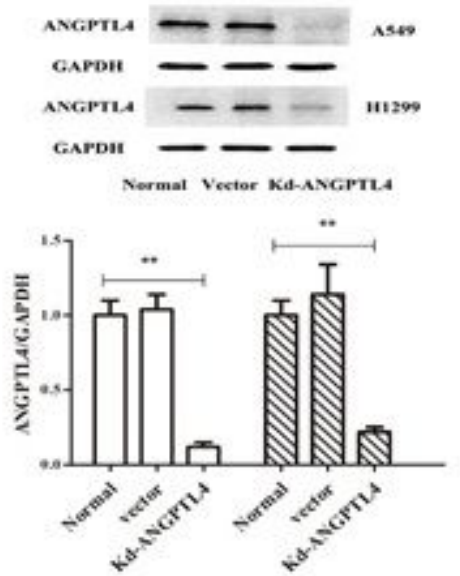

C

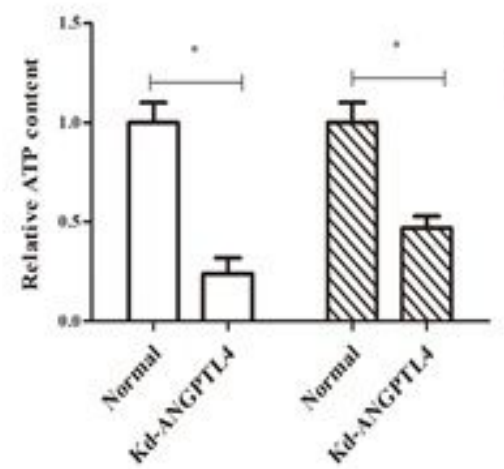

\section{Figure 1}

ANGPTL4 knockdown reduces the proliferation and ATP content of A549 and H1299 cells (A)The viability of normal NSCLC and ANGPTL4 knockdown cells was analyzed by CCK in A549 cells and H1299 cell. (B) The expression of ANGPTL4 in normal NSCLC and ANGPTL4 knockdown cells were determined by Western blot analysis of protein level. ${ }^{\star \star} P<0.01$. (C) The ATP content in normal NSCLC and ANGPTL4 knockdown cells was analyzed. ${ }^{*} \mathrm{P}<0.05$. 
A
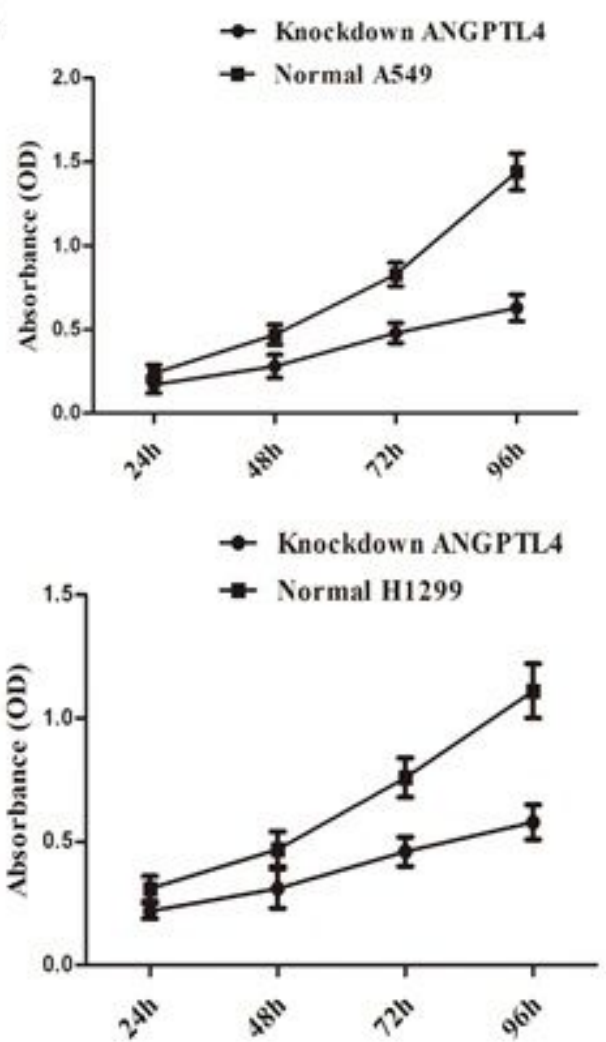

B
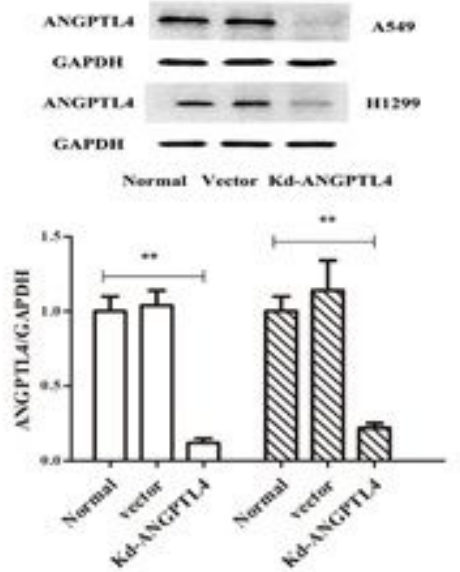

C

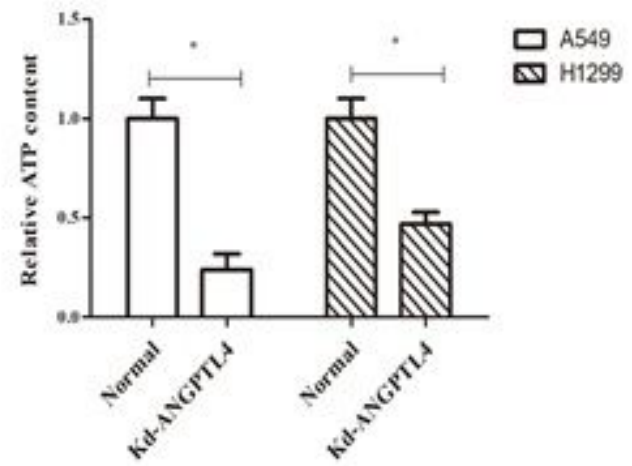

Figure 1

ANGPTL4 knockdown reduces the proliferation and ATP content of A549 and H1299 cells (A)The viability of normal NSCLC and ANGPTL4 knockdown cells was analyzed by CCK in A549 cells and H1299 cell. (B) The expression of ANGPTL4 in normal NSCLC and ANGPTL4 knockdown cells were determined by Western blot analysis of protein level. ${ }^{\star \star} P<0.01$. (C) The ATP content in normal NSCLC and ANGPTL4 knockdown cells was analyzed. ${ }^{*} \mathrm{P}<0.05$.

A
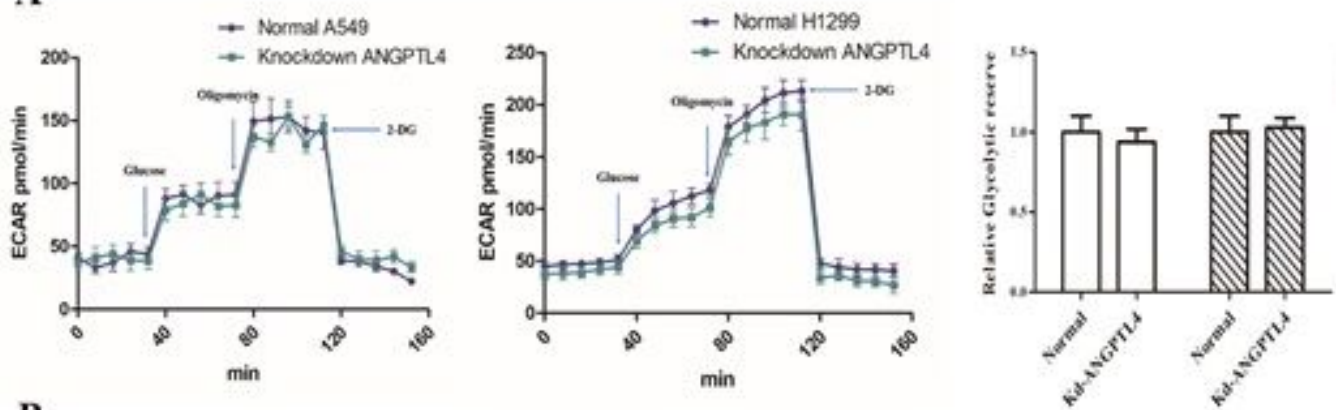

B
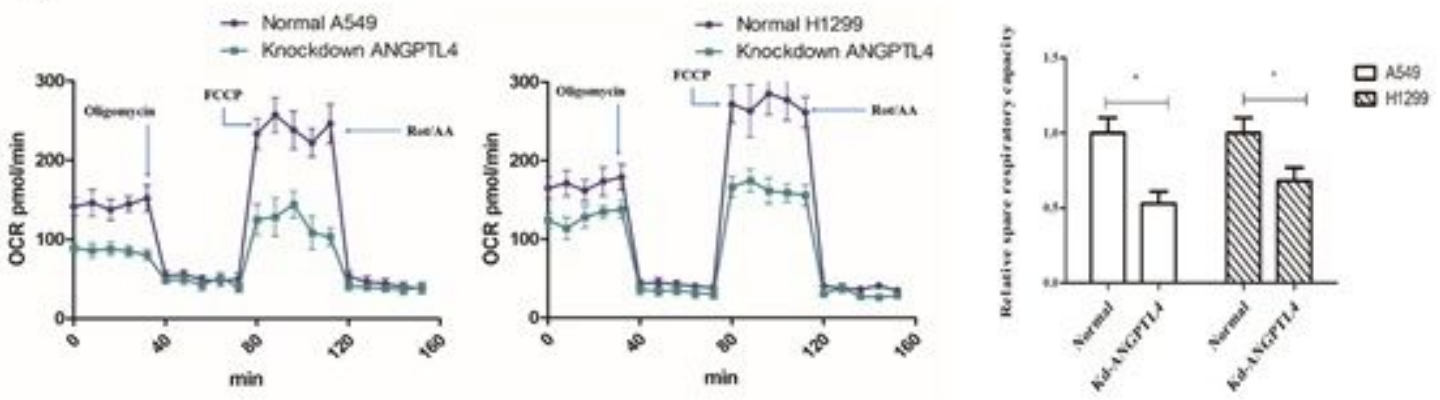


\section{Figure 2}

ANGPTL4 perform OXPHOS but not aerobic glycolysis. (A) Representative trace (left) and mean data (center) of glycolytic ECAR when assayed in minimal medium and treated with glucose in Seahorse. Glycolytic reserve (right) is the difference between glycolytic capacity and glycolysis rate and was measured as the difference between resulting ECAR values after in-Seahorse exposure to glucose and maximal ECAR values obtained after oligomycin treatment. (B) Representative trace (left) and mean data (center) of oxygen consumption rate (OCR) from cells as in a. Values are averages of 5-8 basal OCR measurements minus averages of 3-5 OCR readings after rot/AA treatment of individual wells from indicated cell types. Spare respiratory capacity is the difference between basal OCR values and maximal OCR values obtained after FCCP uncoupling (right). ${ }^{*} P<0.05$, $* * P<0.01,2 D G, 2$-deoxy-d-glucose; FCCP, carbonyl cyanide-4 (trifluoromethoxy) phenylhydrazone; rot/AA, rotenone and antimycin A.
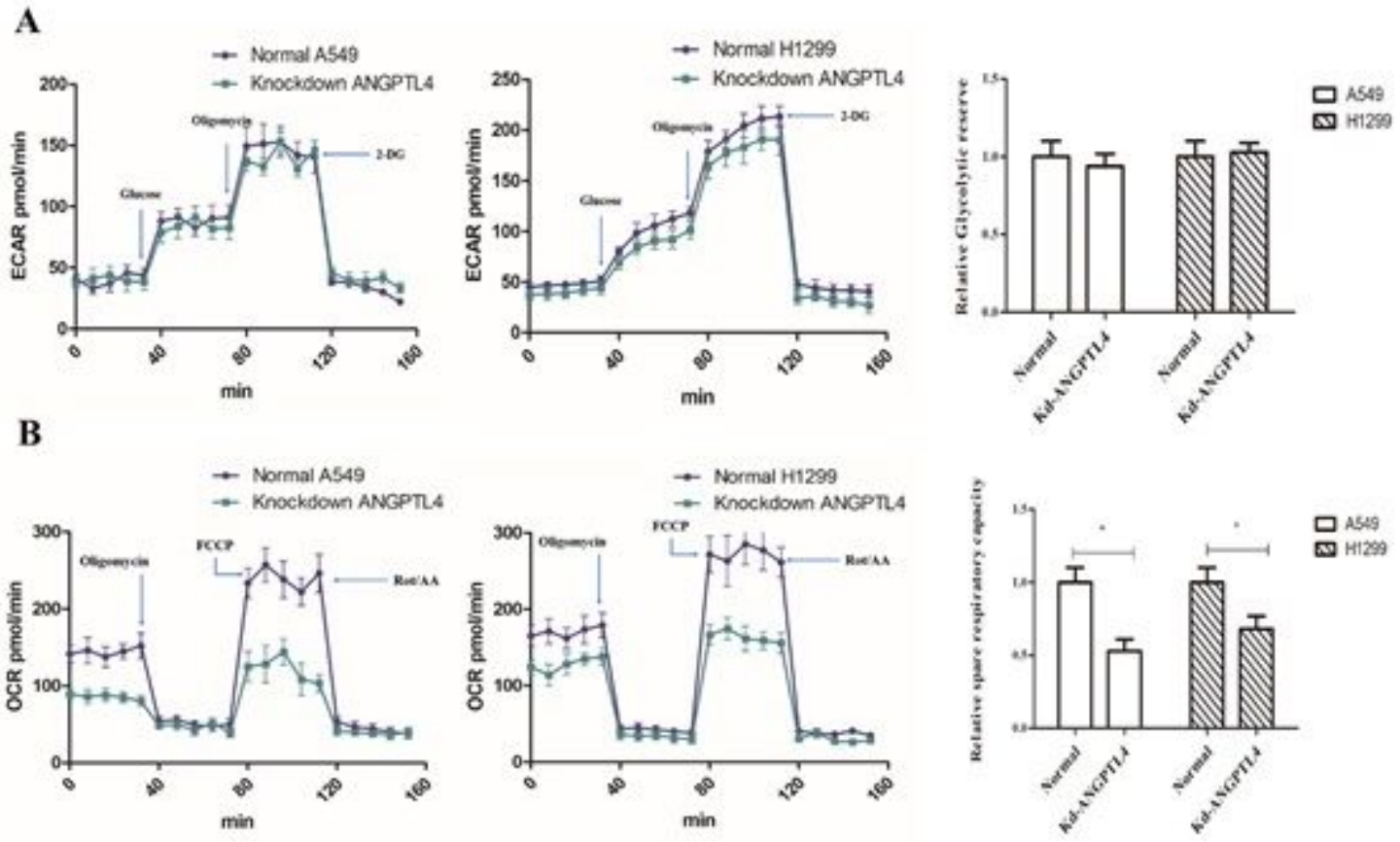

Figure 2

ANGPTL4 perform OXPHOS but not aerobic glycolysis. (A) Representative trace (left) and mean data (center) of glycolytic ECAR when assayed in minimal medium and treated with glucose in Seahorse. Glycolytic reserve (right) is the difference between glycolytic capacity and glycolysis rate and was measured as the difference between resulting ECAR values after in-Seahorse exposure to glucose and maximal ECAR values obtained after oligomycin treatment. (B) Representative trace (left) and mean data (center) of oxygen consumption rate (OCR) from cells as in a. Values are averages of 5-8 basal OCR measurements minus averages of 3-5 OCR readings after rot/AA treatment of individual wells from indicated cell types. Spare respiratory capacity is the difference between basal OCR values and maximal OCR values obtained after FCCP uncoupling (right). ${ }^{*} P<0.05$, $* * P<0.01,2 D G$, 2-deoxy-d-glucose; FCCP, carbonyl cyanide-4 (trifluoromethoxy) phenylhydrazone; rot/AA, rotenone and antimycin A. 


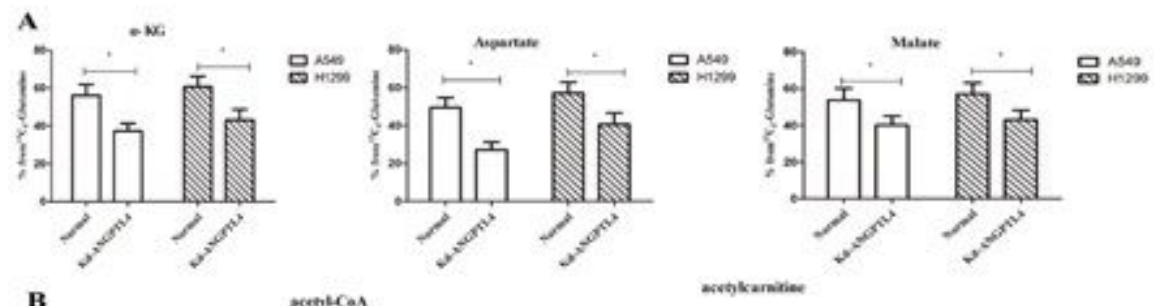

B

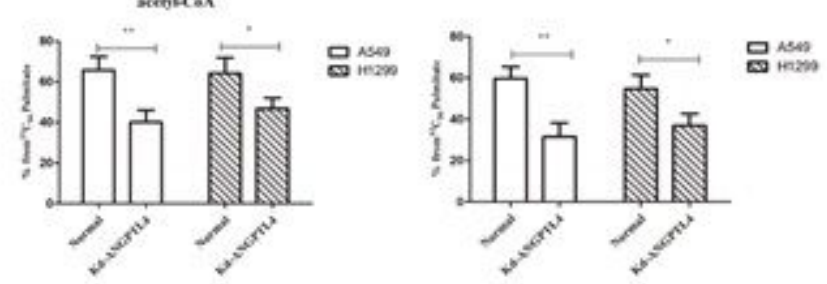

C
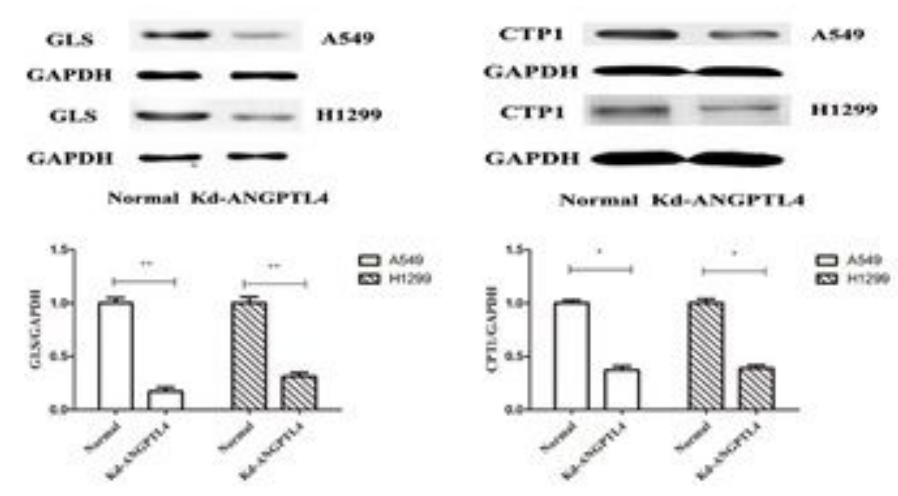

Figure 3

ANGPTL4 promotes glutamine metabolism and fatty acid oxidation. (A) (A) Glutamine consumption determined in NSCLC cells, isotope abundance of a-KG $(M+5)$, malate $(M+4)$, aspartate $(M+4)$ in NSCLC cells were traced by $13 \mathrm{C} 5$-glutamine. ${ }^{*} P<0.05$. (B) Fatty acid oxidation determined in NSCLC cells, the content of [13C16]-acetyl-CoA and [13C16]-acetylcarnitine isotope were traced by 13C16-palmitate. ${ }^{*}<<0.05$. (C) ANGPTL4 regulates glutamine metabolism and fatty acid oxidation in NSCLC cells by inhibiting GLS and CPT1. The expression of GLS and CPT1 in normal NSCLC and ANGPTL4 knockdown cells were determined by Western blot analysis of protein level. ${ }^{*} P<0.01$. ${ }^{*}<<0.05$. 


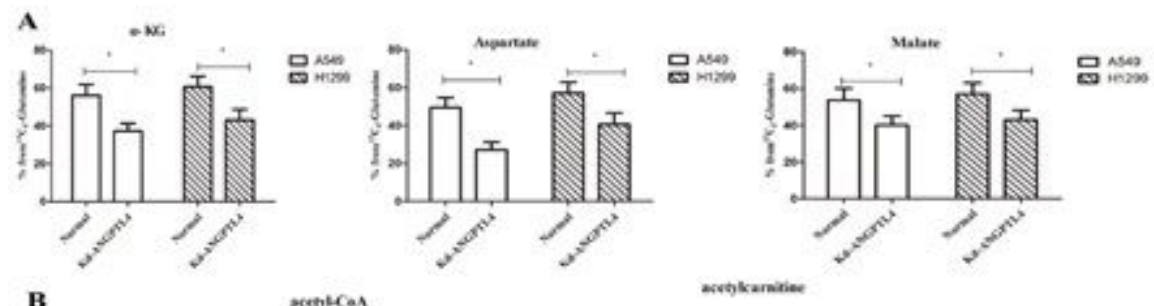

B

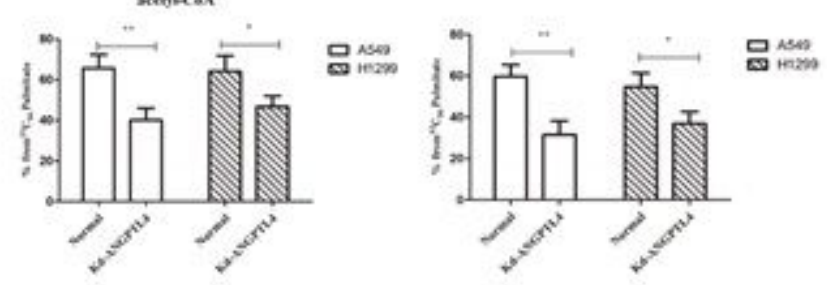

C
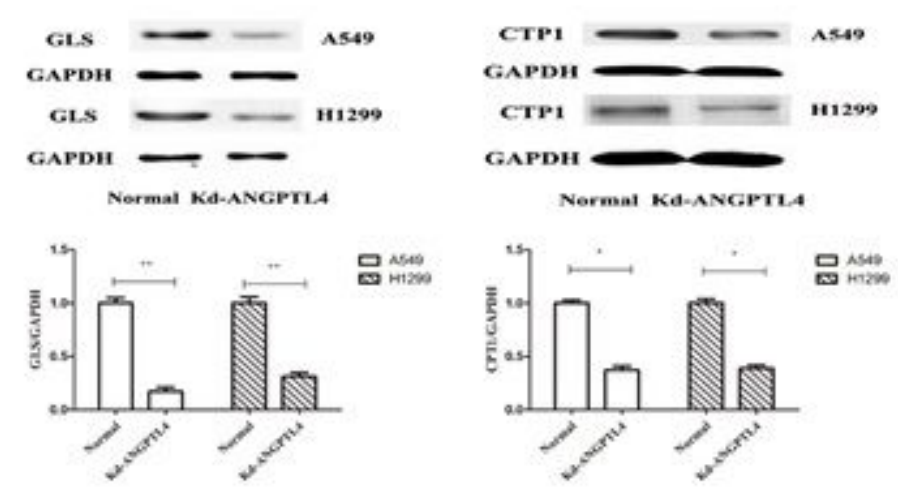

Figure 3

ANGPTL4 promotes glutamine metabolism and fatty acid oxidation. (A) (A) Glutamine consumption determined in NSCLC cells, isotope abundance of a-KG $(M+5)$, malate $(M+4)$, aspartate $(M+4)$ in NSCLC cells were traced by $13 \mathrm{C} 5$-glutamine. ${ }^{*} P<0.05$. (B) Fatty acid oxidation determined in NSCLC cells, the content of [13C16]-acetyl-CoA and [13C16]-acetylcarnitine isotope were traced by 13C16-palmitate. ${ }^{*}<<0.05$. (C) ANGPTL4 regulates glutamine metabolism and fatty acid oxidation in NSCLC cells by inhibiting GLS and CPT1. The expression of GLS and CPT1 in normal NSCLC and ANGPTL4 knockdown cells were determined by Western blot analysis of protein level. ${ }^{*} P<0.01$. ${ }^{*}<<0.05$. 

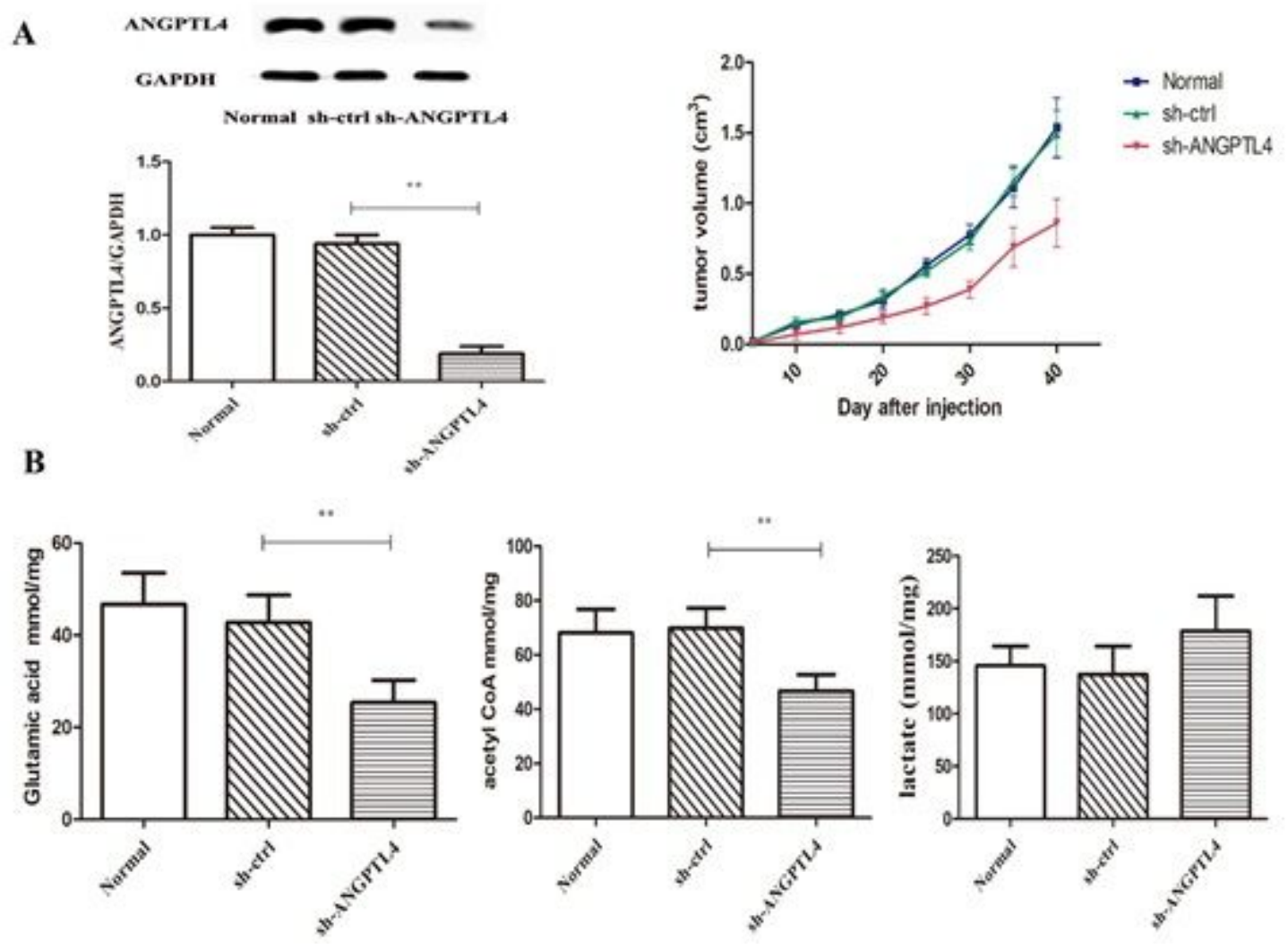

Figure 4

ANGPTL4 knockdown inhibited the tumor growth, glutamine metabolism and fatty acid oxidation in subcutaneous xenograft model. (A). Tumor growth curves of subcutaneous xenograft model for NSCLC cells with treatment as indicated. (B) Glutamic acid, acetyl coa, lactate content were determined in subcutaneous xenograft model. ${ }^{*} \mathrm{P}<0.01$. 

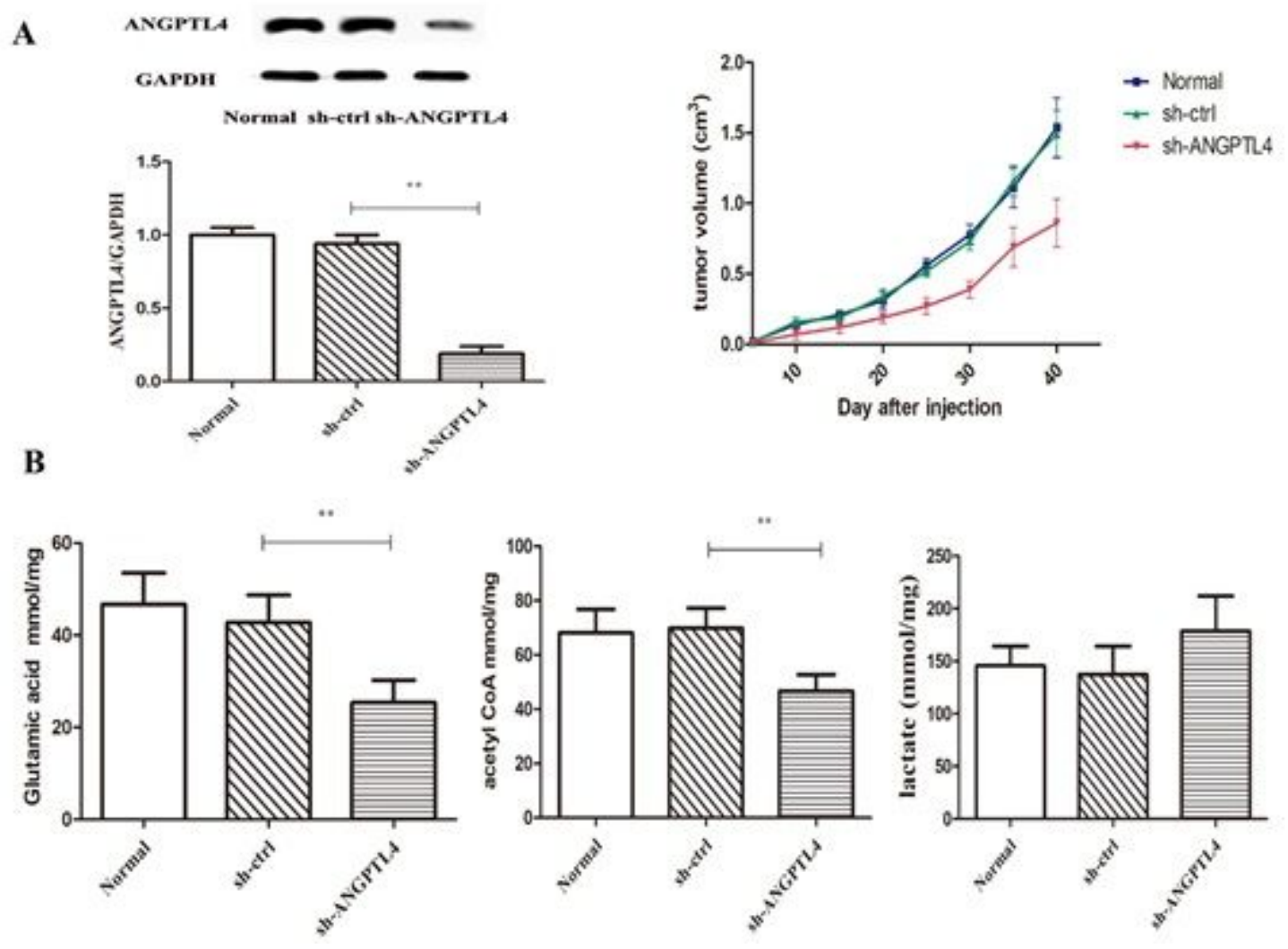

Figure 4

ANGPTL4 knockdown inhibited the tumor growth, glutamine metabolism and fatty acid oxidation in subcutaneous xenograft model. (A). Tumor growth curves of subcutaneous xenograft model for NSCLC cells with treatment as indicated. (B) Glutamic acid, acetyl coa, lactate content were determined in subcutaneous xenograft model. ${ }^{*} \mathrm{P}<0.01$. 
A

Gorrt

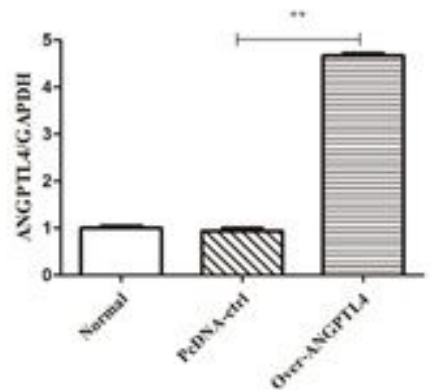

- Plsam etri

- Noreal $\mathrm{Nes}$

* Overespressioa AVGPTLA

- Overespression AVGPR.A without

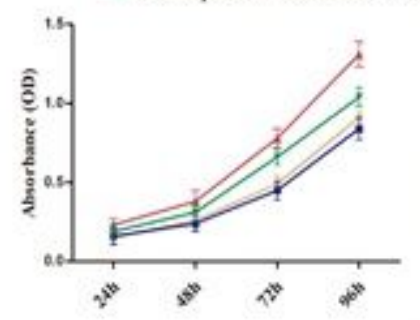

B
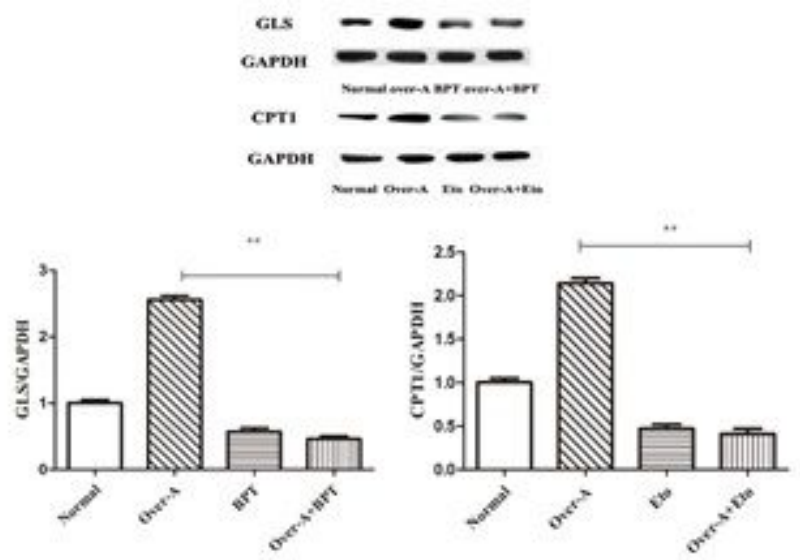

- ON-ANGPTLA

- ON-ANCPTL 4+E10

$\rightarrow$ ON-ANGPTL4+BPTES

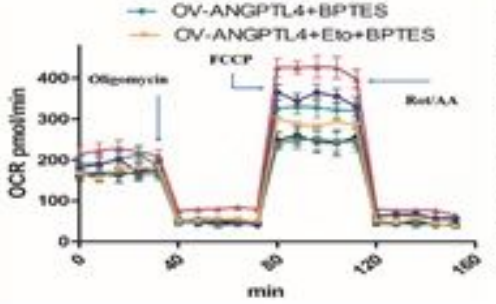

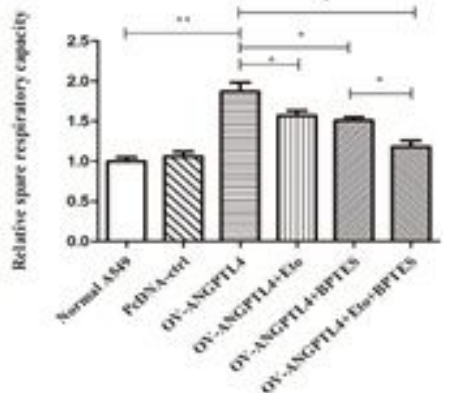

Figure 5

ANGPTL4 overexpression promoted NSCLC proliferation and OXPHOS of A549 cells, but inhibited by CPT1 and GLS inhibitors. (A) ANGPTL4 overexpression promoted NSCLC proliferation, (A)The viability of ANGPTL4 overexpression cells in normal environmental and without glutamine was analyzed by CCK in A549 cells. (B) Representative trace and mean data (center) of oxygen consumption rate (OCR) from ANGPTL4 overexpression cells with CPT1 inhibitor (ETO) or GLS inhibitor(BPTES) were determined. Results are representative of (for sample data) or represent the mean of 3-6 independent experiments. *P $<0.05$, $* *$ P $<0.01,2 D G, 2$-deoxy-d-glucose; FCCP, carbonyl cyanide-4 (trifluoromethoxy) phenylhydrazone; $\mathrm{rot} / \mathrm{AA}$, rotenone and antimycin $\mathrm{A}$. 
A
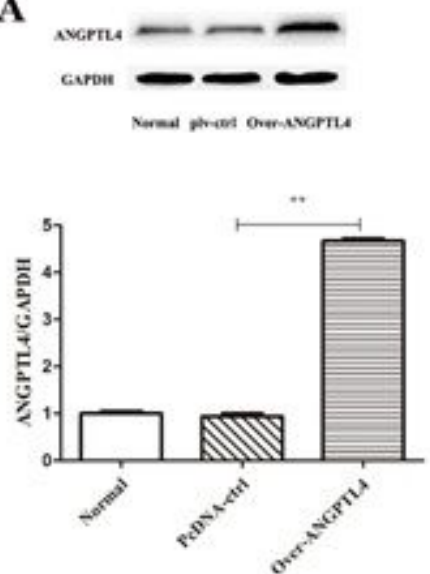

- Plam etrl

- Normal $\mathrm{Nes}$

* Overespressioa AVGPTL4

- Overespression AVGPI.4 witbout

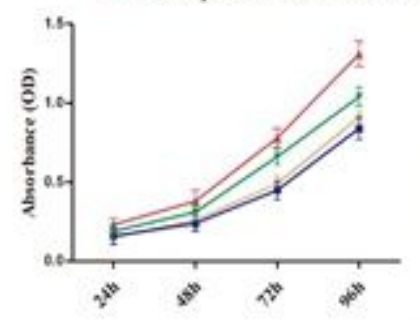

B
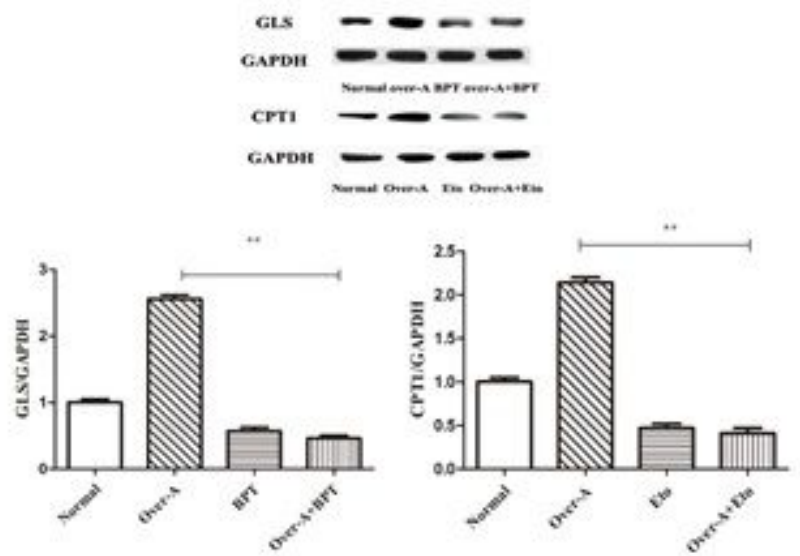

$\div$ OV-ANGPTLA

- ON-ANCPTL 4+E10

- ON-ANGPTL4+BPTES

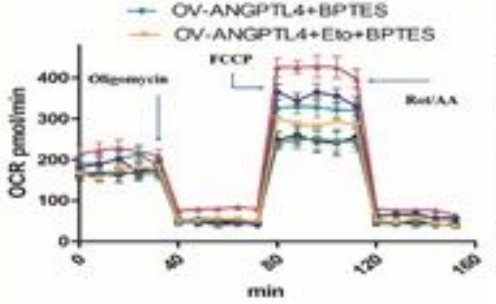

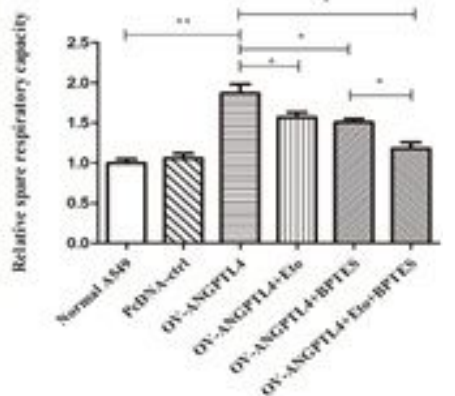

Figure 5

ANGPTL4 overexpression promoted NSCLC proliferation and OXPHOS of A549 cells, but inhibited by CPT1 and GLS inhibitors. (A) ANGPTL4 overexpression promoted NSCLC proliferation, (A)The viability of ANGPTL4 overexpression cells in normal environmental and without glutamine was analyzed by CCK in A549 cells. (B) Representative trace and mean data (center) of oxygen consumption rate (OCR) from ANGPTL4 overexpression cells with CPT1 inhibitor (ETO) or GLS inhibitor(BPTES) were determined. Results are representative of (for sample data) or represent the mean of 3-6 independent experiments. *P $<0.05$, $* *$ P $<0.01,2 D G, 2$-deoxy-d-glucose; FCCP, carbonyl cyanide-4 (trifluoromethoxy) phenylhydrazone; $\mathrm{rot} / \mathrm{AA}$, rotenone and antimycin $\mathrm{A}$. 
A
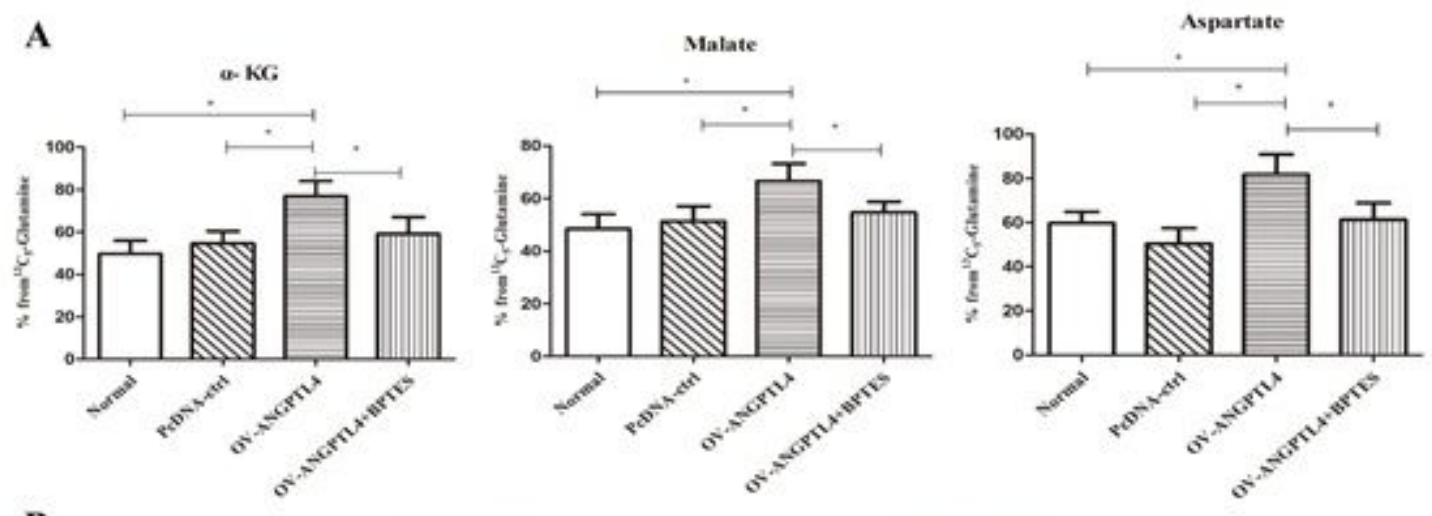

B
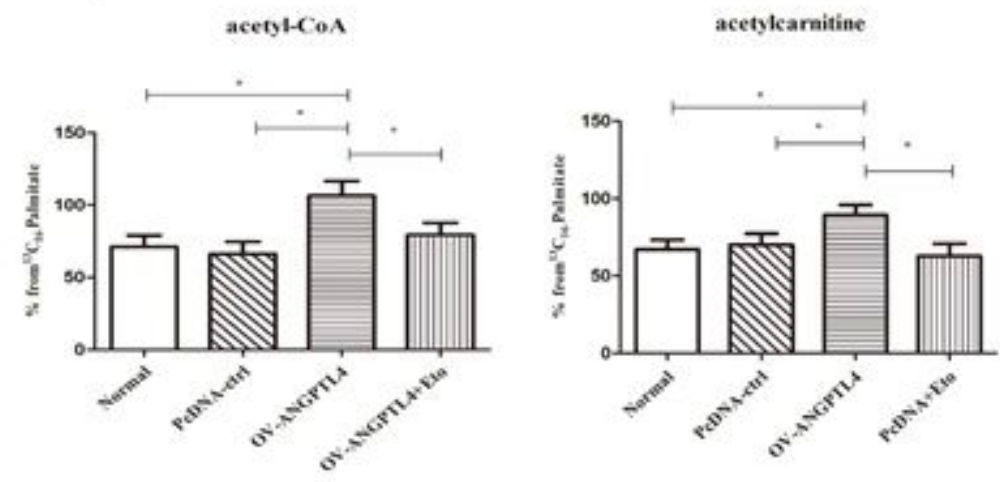

Figure 6

ANGPTL4 overexpression promotes glutamine metabolism and fatty acid oxidation, but inhibited by CPT1 and GLS inhibitors. (A) Glutamine consumption determined in NSCLC cells, isotope abundance of a-KG $(M+5)$, malate $(M+4)$, aspartate $(M+4)$ in NSCLC cells were traced by $13 C 5$-glutamine. ${ }^{*} P<0.05$. (B) Fatty acid oxidation determined in NSCLC cells, the content of [13C16]-acetyl-CoA and [13C16]acetylcarnitine isotope were traced by $13 \mathrm{C} 16$-palmitate. ${ }^{*}<0.05$. NSCLC cell which pretreatment with

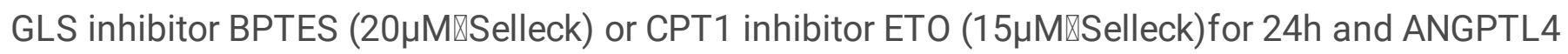
overexpression NSCLC cell combined with BPTES or ETO. *P<0.05. 
A
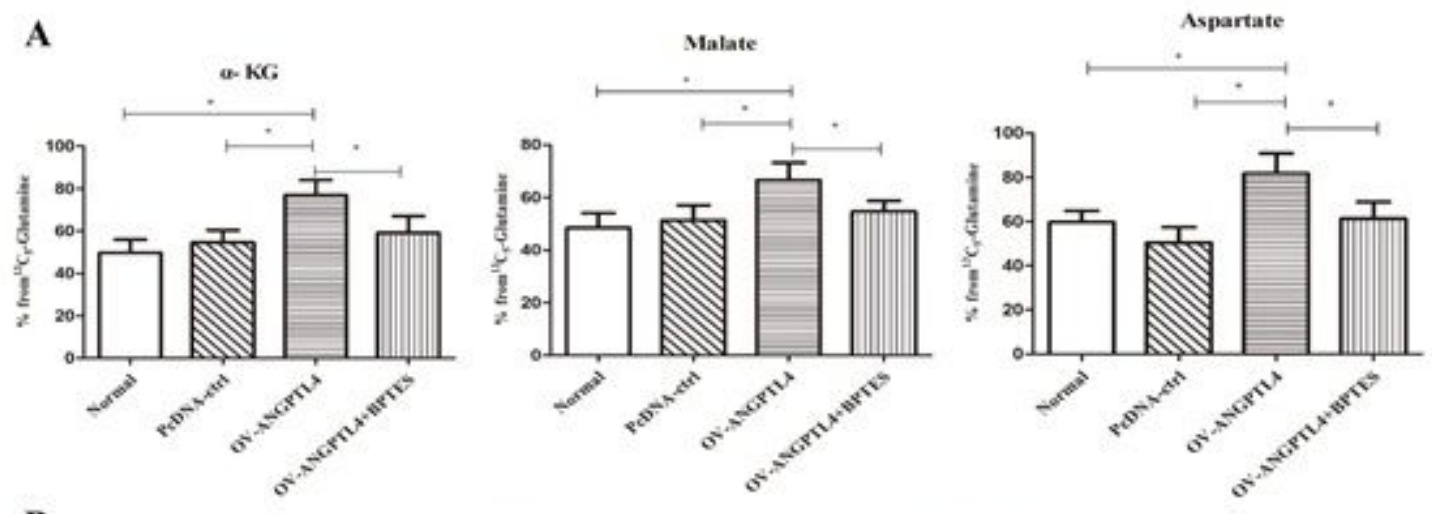

B
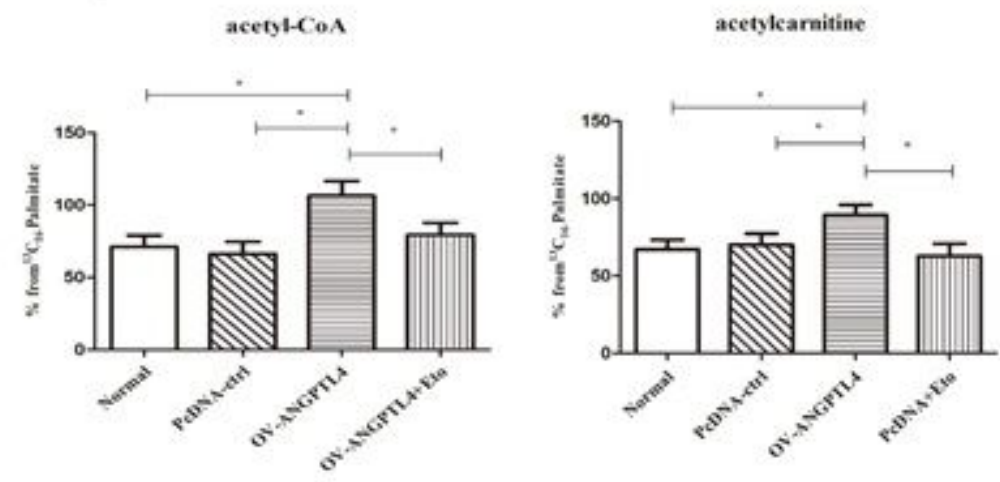

Figure 6

ANGPTL4 overexpression promotes glutamine metabolism and fatty acid oxidation, but inhibited by CPT1 and GLS inhibitors. (A) Glutamine consumption determined in NSCLC cells, isotope abundance of $\mathrm{a}-\mathrm{KG}(\mathrm{M}+5)$, malate $(\mathrm{M}+4)$, aspartate $(\mathrm{M}+4)$ in NSCLC cells were traced by $13 \mathrm{C} 5$-glutamine. ${ }^{*} \mathrm{P}<0.05$. (B) Fatty acid oxidation determined in NSCLC cells, the content of [13C16]-acetyl-CoA and [13C16]acetylcarnitine isotope were traced by $13 \mathrm{C} 16$-palmitate. ${ }^{*} \mathrm{P}<0.05$. NSCLC cell which pretreatment with

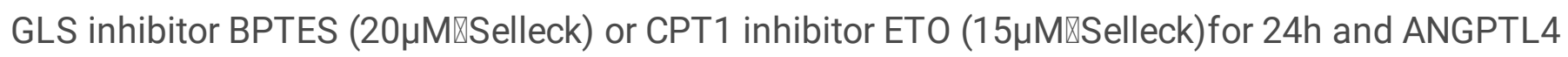
overexpression NSCLC cell combined with BPTES or ETO. *P<0.05.

\section{Supplementary Files}

This is a list of supplementary files associated with this preprint. Click to download.

- supplementdata.docx

- supplementdata.docx 